\title{
Influences of Network Delay on Quality of Experience for Soft Objects in Networked Real-Time Game with Haptic Sense
}

\author{
Mya Sithu', Yutaka Ishibashi' ${ }^{1}$ Pingguo Huang' ${ }^{2}$, Norishige Fukushima1 \\ ${ }^{1}$ Graduate School of Engineering, Nagoya Institute of Technology, Nagoya, Japan \\ ${ }^{2}$ Faculty of Engineering Division II, Tokyo University of Science, Tokyo, Japan \\ Email:s.mya.492@stn.nitech.ac.jp,ishibasi@nitech.ac.jp,huang@ms.kagu.tus.ac.jp, \\ fukushima@nitech.ac.jp
}

Received 23 September 2015; accepted 15 November 2015; published 18 November 2015

Copyright (C) 2015 by authors and Scientific Research Publishing Inc.

This work is licensed under the Creative Commons Attribution International License (CC BY). http://creativecommons.org/licenses/by/4.0/

(c) () D Den Access

\begin{abstract}
In this paper, we investigate the influences of network delay on QoE (Quality of Experience) such as the operability of haptic interface device and the fairness between players for soft objects in a networked real-time game subjectively and objectively. We handle a networked balloon bursting game in which two players burst balloons (i.e., soft objects) in a 3D virtual space by using haptic interface devices, and the players compete for the number of burst balloons. As a result, we find that the operability depends on the network delay from the local terminal to the other terminal, and the fairness is mainly dependent on the difference in network delay between the players' terminals. We confirm that there exists a trade-off relationship between the operability and the fairness. We also see that the contribution of the fairness is larger than that of the operability to the comprehensive quality (i.e., the weighted sum of the operability and fairness). Assessment results further show that the output timing of terminals should be adjusted to the terminal which has the latest output timing to maintain the fairness when the difference in network delay between the terminals is large. In this way, the comprehensive quality at each terminal can be maintained as high as possible.
\end{abstract}

\section{Keywords}

Networked Real-Time Game, Virtual Environment, Balloon Bursting Game, Haptic Interface Devices, Network Delay, Quality of Experience, Operability, Fairness

\section{Introduction}

A number of researchers have been paying their attention to networked real-time games with haptic sense [1]-[6].

How to cite this paper: Sithu, M., Ishibashi, Y., Huang, P.G. and Fukushima, N. (2015) Influences of Network Delay on Quality of Experience for Soft Objects in Networked Real-Time Game with Haptic Sense. Int. J. Communications, Network and System Sciences, 8, 440-455. http://dx.doi.org/10.4236/ijcns.2015.811040 
Players can perceive a higher sense of immersion by using haptic interface devices in networked real-time games. When such a game is played over a network where QoS (Quality of Service) [7] is not guaranteed like the Internet, the consistency (e.g., the positions of an object at different terminals are the same) of objects in a $3 \mathrm{D}$ virtual space at the players' terminals may be disturbed owing to the network delay, delay jitter, and packet loss.

To keep the consistency high at the terminals, we can employ the local lag control [5], which buffers the local information for a constant time called the local lag according to the network delay from the local terminal to the other terminal. Thus, the interactivity is degraded when the local lag is large. The operability of haptic interface device may also be deteriorated. If the network delay from the local terminal to the other terminal is about the same to that from the other terminal to the local terminal, the fairness is not deteriorated although the operability is deteriorated. However, when the difference in network delay between the terminals is large, the fairness among the players is largely damaged [6]. For example, in a networked real-time game when two players play, when the network delay from a terminal to the other terminal is large, and that in the opposite direction is small, the operability is seriously degraded only at the terminal with the larger network delay under the local lag control. This leads to unfairness between the players. In [8], Brun et al. described that the fairness was high when the same condition was provided to all the players.

To maintain the fairness high, we can use the adaptive $\Delta$-causality control [9], which also employs the local lag control. The adaptive $\Delta$-causality control generally sets the local lag at each terminal to the maximum network delay among the terminals. Therefore, when the maximum network delay is large, the interactivity may seriously be degraded under the control. This leads to the severe degradation of the operability of the haptic interface device, but the fairness is maintained high. Based on this relationship of the operability and fairness, we can say that there is a trade-off relationship between the operability and fairness.

In previous works, for example [3] [4], the influence of network delay on the fairness is investigated using hard objects in a 3D virtual space. In [3], a networked real-time game in which each of two players tries to contain a target (a sphere) competitively with his/her object (a rigid cube) by manipulating his/her haptic interface device is dealt with in a 3D virtual space. Assessment results show that the players feel unfairness when the difference in network delay between them is larger than about $30 \mathrm{~ms}$. Also, in [4], the influence of the time it takes for a smell to reach a player on the fairness is investigated in a fruit harvesting game where two players play in a 3D virtual space. In the game, all the fruit objects in the 3D virtual space are hard ones. As a result, it is illustrated that the fairness is hardly damaged when the time is smaller than about $500 \mathrm{~ms}$.

In [3] and [4], since only the fairness is investigated, the relationship between the operability of haptic interface device and the fairness between players is not clear. It is important to investigate the relationship of the operability and fairness in detail by QoE (operability and fairness) [10] assessment in which we should assess the comprehensive quality (i.e., the weighted sum of the operability and fairness) to examine which QoE has larger contribution to the comprehensive quality. Also, we should clarify how much local lag should be set at each terminal to maintain the comprehensive quality as high as possible. For example, if the fairness has larger contribution to the comprehensive quality, the comprehensive quality may be maintained high by adjusting the output timing of the terminals to the terminal which has the slowest timing as in the adaptive $\Delta$-causality control. Furthermore, to the best of our knowledge, there is no previous work which investigates the influence of network delay on QoE such as the operability and fairness for soft objects in virtual environments. In the case of hard objects, it is found that the objects become heavier as the network delay increases [11]; that is, their characteristics change owing to network delay. The characteristics of soft objects may change in the same way as those of hard objects; for example, they become harder and/or heavier as the network delay increases. However, it is not clear how the characteristics change. We should carry out QoE assessments for soft objects to understand clearly how their characteristics change when the network delay increases.

In this paper, we investigate the influences of network delay on the operability of the haptic interface device and the fairness between players by carrying out QoE assessments subjectively in a balloon bursting game, where two players burst balloons (i.e., soft objects) in a 3D virtual space. We also perform objective QoE assessment at the same time as the subjective assessment. We further investigate the relationship between subjective and objective assessment results.

\section{Balloon Bursting Game}

\subsection{System Configuration}

The system configuration of the balloon bursting game is shown in Figure 1, where each of two players (players 


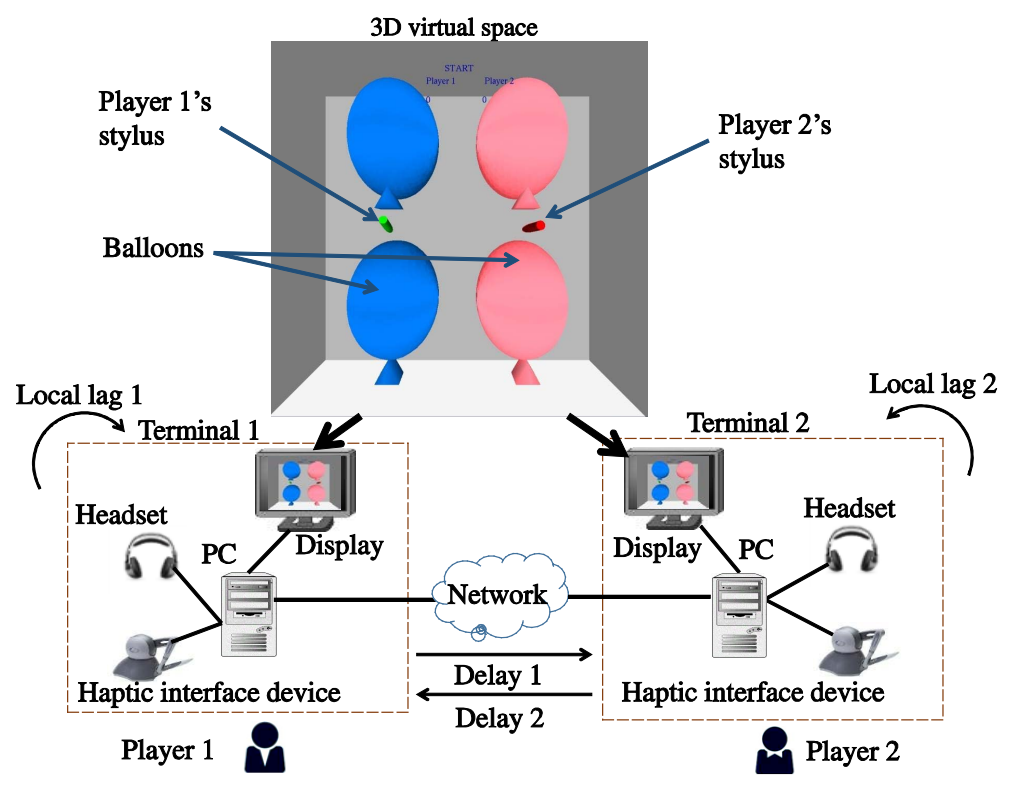

Figure 1. System coniguration of balloon bursting game.

1 and 2) bursts balloons with his/her respective stylus in a 3D virtual space, and the two players compete with eachother for the number of burst balloons. The system consists of two terminals (terminals 1 and 2), each of which has a PC, a haptic interface device (Geometric Touch [12]), a display, and a headset. A player at each terminal employs the haptic interface device to move the virtual stylus (a CG image of the stylus of the haptic interface device) in the virtual space. When the player touches the balloon with the tip of the stylus, the reaction force is perceived through the haptic interface device; he/she can feel the softness of the balloon. The balloon is distorted when the player pushes the balloon with the stylus. If he/she pushes it strongly, the balloon is largely distorted, and it is burst and disappeared. Then, he/she hears a sound of bursting it via the headset.

In addition, we enhance the balloon bursting game by adding a function handling the olfactory sense to carry out an investigation as in [4]. We are also investigating the influence of network delay on the fairness by using the enhanced application with olfactory and haptic senses [13]. It is also important to carry out experiments in the enhanced application by assessing both operability and fairness like the work in this paper. However, this is for further study.

\subsection{Playing Method}

In this paper, as shown in Figure 1, there are four balloons in the 3D virtual space. Player 1 bursts two blue balloons alternately on the left side of the virtual space, and player 2 bursts two pink balloons on the right side. This purpose is to avoid the situation of trying to burst the same balloon simultaneously at the two terminals for simplicity. If both players try to burst the same balloon at the same time, we can determine which player bursts the balloon earlier than the other user by using AtoZ [14] and Count Down Protocol [15] as in [16]. Before the start of the game, the players stand ready by placing their styli at their respective original positions (see Figure 2(a)). The players start to burst the balloons when "START" message is displayed on the screen and a buzzer sound is output (see Figure 2(a)). During the game, the numbers of burst balloons of the two players are displayed on the screen (see Figure 2(b)). The players stop the game when "GAME OVER" message appears on the screen 30 seconds after the beginning of the game (see Figure 2(c)). The buzzer sound also alerts the players to stop the game at that time. A player who has burst more balloons than the other player wins the game; player 2 wins in Figure 2(c). When a balloon is burst and disappeared, a new balloon automatically appears at the location of the burst balloon. Both players try to burst their respective balloons from the front side of the balloon as fast as they can.

\subsection{Calculation of Reaction Force}

The reaction force applied to the haptic interface device is generated by the haptic rendering engine [17], which uses the object shape and material properties such as stiffness and friction for calculation of the reaction force. 


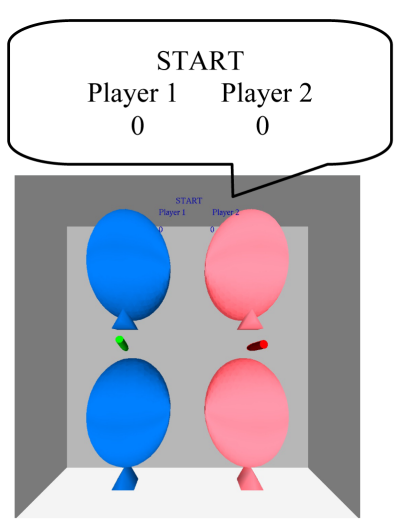

(a)

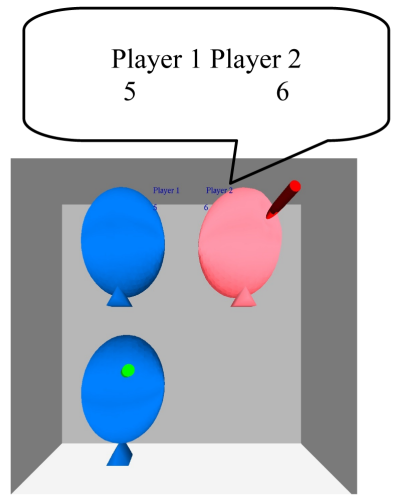

(b)

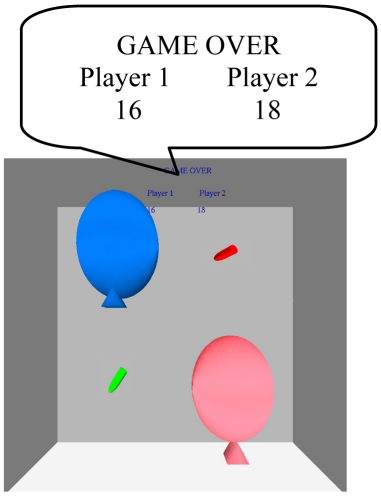

(c)

Figure 2. Displayed images in virtual space. (a) Start of game; (b) During game; (c) End of game.

In order to get the reaction force against a player via the haptic interface device, we use the following function of the Open Haptics HLAPI (Haptic Library Application Programing Interface) [17]:

$$
\text { hlGetDoublev (HL_REACTION_FORCE, r_force), }
$$

where hlGetDoublev() is a function used for getting the reaction force (HL_REACTION_FORCE) from the haptic rendering engine, and r_force is a user-defined parameter which receives a returned value of HL_REACTION_FORCE. The value of the force applied to a balloon when a player pushes the balloon with a stylus is received from the parameter HL_CURRENT_FORCE. In a preliminary experiment, we confirmed that the value of HL_CURRENT_FORCE is almost equal to that of HL_REACTION_FORCE; that is, a player feels almost the same reaction force to the force pushed by him/her. The player feels larger reaction force as the penetration depth of the stylus becomes larger. The penetration depth of the stylus is the distance from the surface of the balloon to the tip of the stylus. When the balloon is distorted by the stylus, its volume is varied. We can obtain the volume from the haptic rendering engine.

In order to clarify the relations among the reaction force, the penetration depth, and the volume of a balloon, we here explain four cases (called the standard balloon case, small balloon case, hard balloon case, and soft balloon case). In the standard balloon case, the radii of three dimensional axes $(x, y$, and $z)$ of the balloon are 1.1, 1.5 and 1.1, respectively (see Figure 3(a)), where we assume that the length of the stylus is 1.0 [18]. In the small balloon case, we use a balloon which has the radii of 0.35, 0.48, and 0.35, respectively (see Figure 3(b)) [18]. Balloons in the hard and soft balloon cases are two times harder and softer, respectively, than those in the standard balloon case; the sizes of the balloons are the same as that in the standard balloon case. In Figures 4(a)-(d) [18], we plot the reaction force, penetration depth, and volume versus the elapsed time for the four cases. The elapsed time is defined as the time interval from the moment the surface of a balloon is touched with the stylus until the instant the balloon is burst. In Figure 5 [18], we show displayed images of a balloon for the standard balloon case when the balloon is distorted; Figures 5(a)-(c) corresponds to the standard balloon at the elapsed times of $500 \mathrm{~ms}, 1000 \mathrm{~ms}$, and $1500 \mathrm{~ms}$, respectively, in Figure 4(b).

\subsection{Judgment of Bursting}

There may be several methods of judgment of bursting a balloon in a virtual space. For example, a balloon can be burst according to the volume of the balloon and/or the force pushed with the stylus. In the former method, the balloon is burst when the volume of the balloon is smaller than a threshold value. In a preliminary experiment where we used the former method, we set the threshold value to $90 \%$ of the initial volume of the balloon.

In the latter method, when the force applied to the balloon from the cursor of the haptic interface device is larger than another threshold value, the balloon is burst. We set the threshold value to $0.4 \mathrm{~N}$ in our preliminary experiment.

As the other method, if either of the conditions of the above methods is met, the balloon can be burst. In our assessment in this paper, we use a method in which a balloon is burst when the volume of a balloon reaches a threshold value for simplicity. 


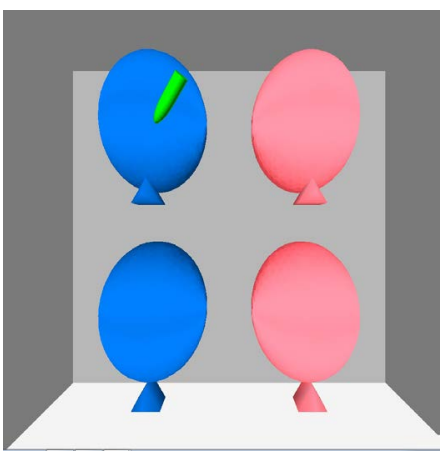

(a)

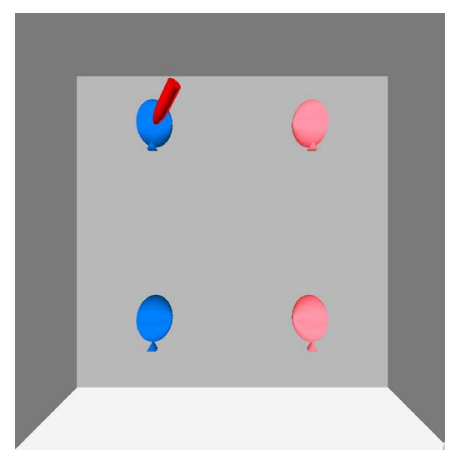

(b)

Figure 3. Displayed images of balloons in standard, small, hard, and soft balloon cases. (a) Standard, hard, and soft balloon cases; (b) Small balloon case.

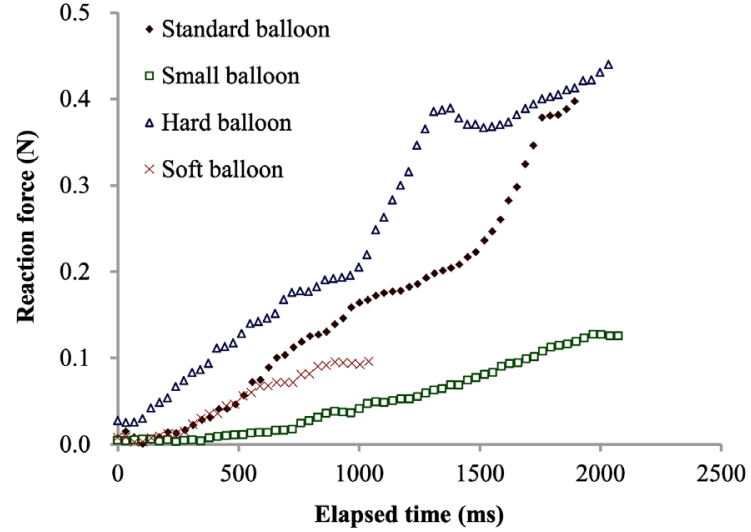

(a)

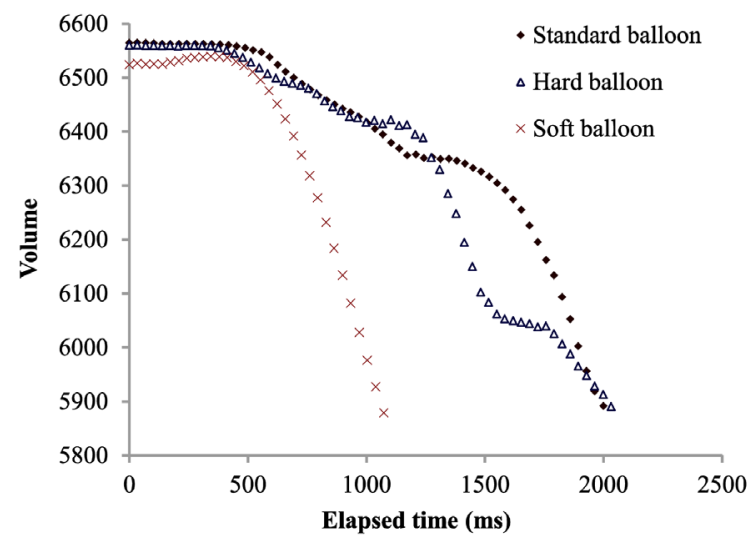

(c)

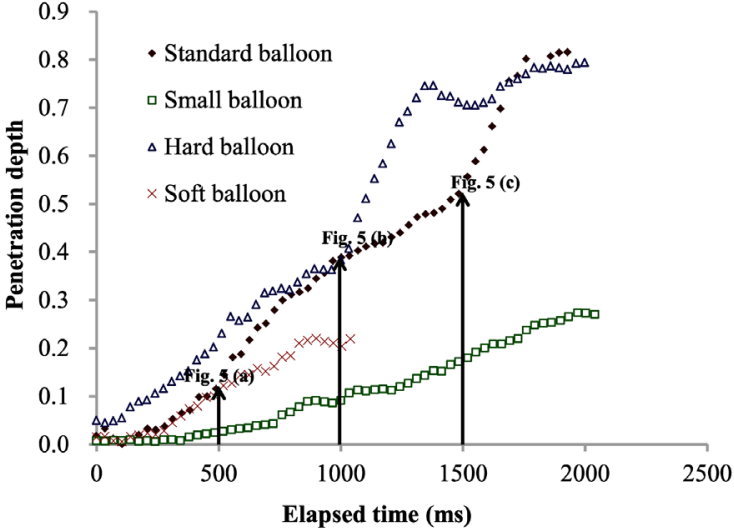

(b)

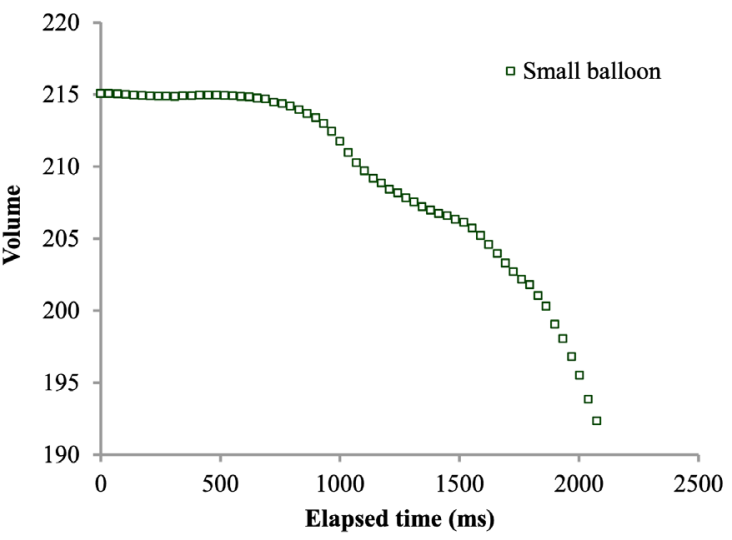

(d)

Figure 4. Relations among reaction force, penetration depth, and volume of balloon. (a) Reaction force; (b) Penetration depth to balloon; (c) Volume of balloon in standard, hard, and soft balloon cases; (d) Volume of balloon in small balloon case.

\section{Assessment Environment}

\subsection{Assessment System}

In our assessment system, the two terminals are connected to each other via a network emulator (NIST Net [19]) which is used instead of the network shown in Figure 1. The network emulator generates an additional constant 


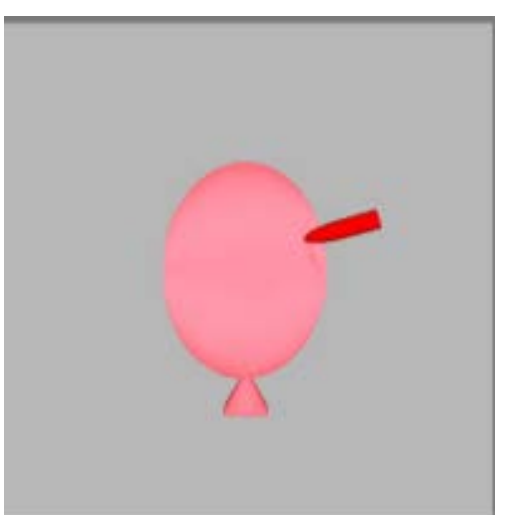

(a)

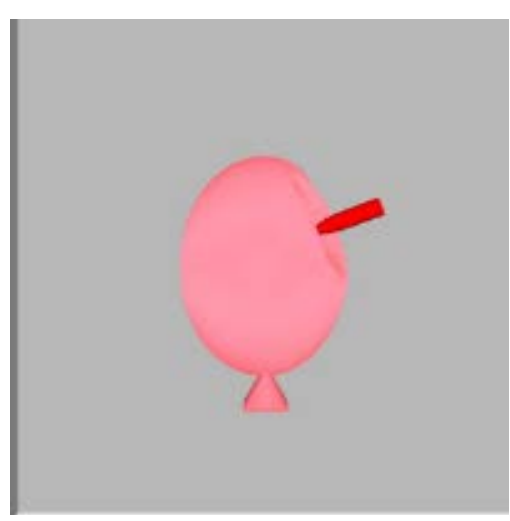

(b)

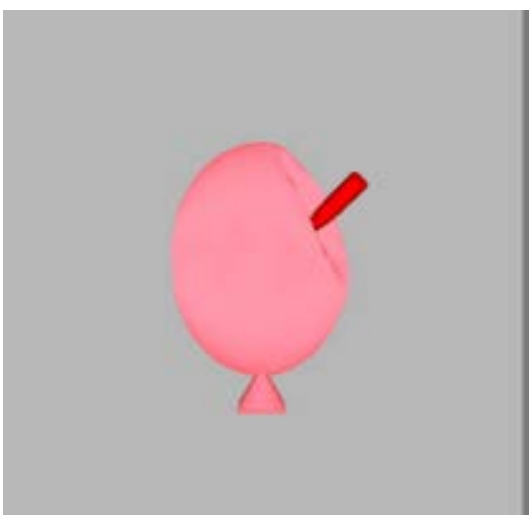

(c)

Figure 5. Displayed images of distorted balloon. (a) Elapsed time: 500 ms; (b) Elapsed time:1000 ms; (c) Elapsed time: $1500 \mathrm{~ms}$.

delay for each packet transmitted between the terminals. We can here take account of network delay jitter as in [20]. We call the constant delay from terminal 1 to 2 delay 1, and that from terminal 2 to 1 delay 2 (see Figure 1). We call the local lag at terminal 1 local lag 1 and that at terminal 2 local lag 2 . There are two cases in our assessment (called the same delay case and the different delay case). In the same delay case, the constant delay from terminal 1 to terminal 2 is always equal to that in the opposite direction. In the different delay case, the constant delay from terminal 1 to terminal 2 can be different from that in the opposite direction.

\subsection{Assessment Methods}

\subsubsection{Same Delay Case}

In this assessment, we investigated the influence of network delay on only the operability of haptic interface device. We did not investigate the fairness between players because the fairness is perfect when the values of network delay are the same between the terminals [6]. In order to focus on the relation between the network delay and the operability of haptic interface device, we only used terminal 1 in this case. Local lag 1 was set to the same values as delay 1. We carried out QoE assessment with 16 subjects (males and females) whose ages were between 20 and 30. Each subject practiced about two minutes under the condition that the constant delays were set to 0 ms before the assessment for each case. In the assessment, each subject burst the two blue balloons alternately which were located on the left side of the virtual space. When a balloon was burst, a new balloon always automatically appeared for the burst balloon. In order to investigate the influence of network delay on the different types of balloons, we carried out four assessments using four types of balloons which are described in Subsection 2.3. The assessments in this case are called the standard balloon assessment, small balloon assessment, hard balloon assessment, and soft balloon assessment, respectively, according to the types of balloons that we use.

In each assessment, we changed the delays in random order from $0 \mathrm{~ms}$ to $500 \mathrm{~ms}$ at intervals of $50 \mathrm{~ms}$ for each subject. The order of assessments was also selected in random order for each subject. The subject burst the balloons with the stylus continuously for 30 seconds. After each stimulus, he/she was asked to base his/her judgment about the easiness of bursting based on the five-grade impairment scales (5: Imperceptible, 4: Perceptible, but not annoying, 3: Slightly annoying, 2: Annoying, 1: Very annoying) [21]. By averaging the scores of all the subjects, we obtained Mean Opinion Score (MOS) [21]. As objective assessment measure, we adopted the number of burst balloons. The total assessment time for each case was about 20 minutes per subject.

\subsubsection{Different Delay Case}

In this case, we carried out QoE assessment to clarify the influences of network delay on the operability, fairness, and comprehensive quality. In the assessment, two subjects played the balloon bursting game together. Before the assessment, each pair of subjects played the balloon bursting game for three times to get used to the game on the condition that delays 1 and 2 are set to $0 \mathrm{~ms}$; that is, the same condition is provided to the pair. By practicing, each subject knows how to burst a balloon by using a haptic interface device. In this case, we carried out the assessment by setting delay 1 to $0 \mathrm{~ms}$ or $200 \mathrm{~ms}$, and setting delay 2 to $50 \mathrm{~ms}, 100 \mathrm{~ms}, 200 \mathrm{~ms}, 300 \mathrm{~ms}$, or $500 \mathrm{~ms}$. 
We carried out QoE assessment with 16 subjects (males and females) whose ages were between 20 and 30 .

Assessments in which delay 1 was set to $0 \mathrm{~ms}$ and $200 \mathrm{~ms}$ are referred to as assessments 1 and 2, respectively. In order to know the suitable local lag values at the two terminals to maintain both operability and fairness, we carried out the assessments by setting local lags 1 and 2 to different values. Local lag 1 was changed from $0 \mathrm{~ms}$ to $500 \mathrm{~ms}$ at intervals of $50 \mathrm{~ms}$, and local lag 2 was set to the same value as delay 2. In each assessment, the order of combinations of delays and the local lags were changed in random order for the pair. It took 30 seconds for each stimulus. After each stimulus, each subject was asked to base his/her judgment about the operability, fairness, and comprehensive quality in terms of wording used to define the five-grade impairment scale (5: Imperceptible, 4: Perceptible, but not annoying, 3: Slightly annoying, 2: Annoying, 1: Very annoying) [21]. In each stimulus, if the pair obtained almost the same results about victory or defeat as those in the practice, the pair regarded the fairness as high and valued the score at 5 . The comprehensive quality is the weighted sum of the operability and interactivity; thus, the comprehensive quality is the most important. Each subject gave a score from 1 through 5 to each stimulus. By averaging the scores of all the subjects, we obtained MOS. We also adopted the number of burst balloons as an objective assessment measure. The total assessment time for each assessment was about two hours per pair.

\section{Assessment Results}

\subsection{Same Delay Case}

We show the MOS values of operability versus delay 1 for four assessments in Figure 6 [18] [22]. We also show the average number of burst balloons versus delay 1 in Figure 7 [18] [22]. In the figures, the 95\% confidence levels are also plotted.

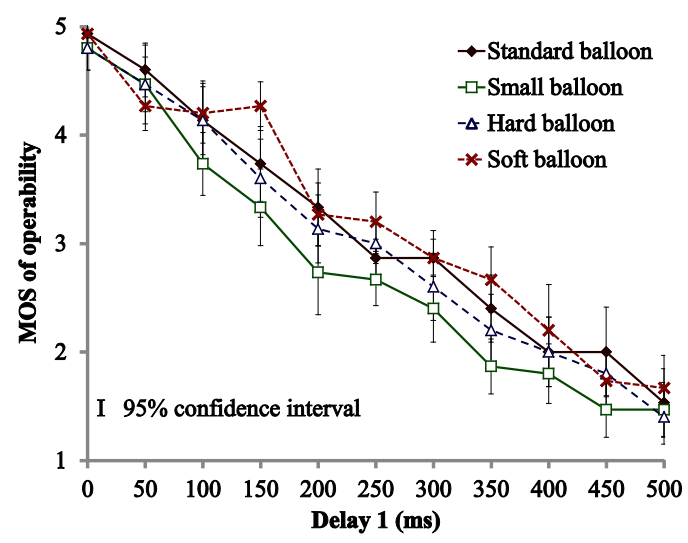

Figure 6. MOS of operability in same delay case.

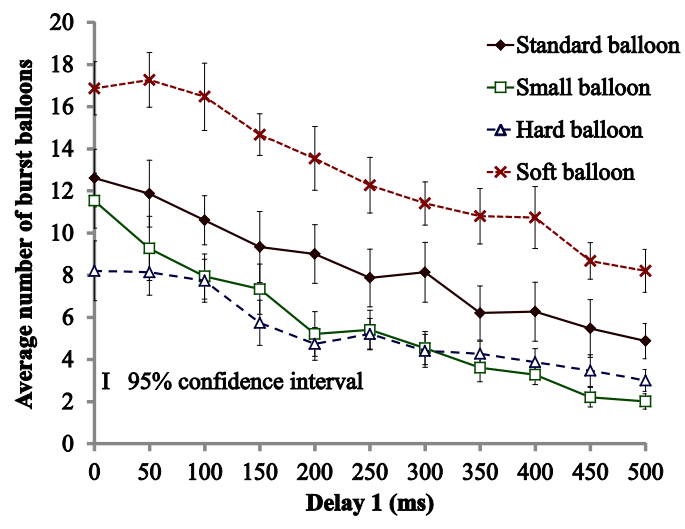

Figure 7. Average number of burst balloons in same delay case. 
In Figure 6, we see that the MOS values of all the assessments decrease as delay 1 becomes larger. This is because the local information at terminal 1 is buffered for a time of delay 1 ; thus, since the interactivity is degraded, each subject feels that the balloon becomes harder and more slippery, and it is difficult to burst the balloon. From the figure, we also find that the MOS value of the small balloon assessment is smaller than those of the other cases when delay 1 is larger than or equal to about $100 \mathrm{~ms}$. The reason is that it is more difficult to operate a virtual stylus so as not to slip from the small balloon when the delay 1 is large. Moreover, we observe in the figure that when delay 1 is smaller than or equal to about $150 \mathrm{~ms}$, the MOS value is higher than 3.5; this means that the deterioration in QoE is allowable for network delays smaller than or equal to about $150 \mathrm{~ms}$ according to [23].

Figure 7 reveals that the average numbers of burst balloons in all the assessments become smaller as delay 1 increases. Form the figure, we also observe that the average number of burst balloons in the soft balloon assessment is the highest. The average number of burst balloons in the hard balloon assessment is the smallest when delay 1 is smaller than around $250 \mathrm{~ms}$. When delay 1 is larger than about $300 \mathrm{~ms}$, the number of burst balloons in the small balloon assessment is the smallest.

\subsection{Different Delay Case}

\subsubsection{Subjective Assessment Results}

We show the MOS values of operability at terminals 1 and 2 as a function of local lag 1 in Figure 8 and Figure 9, respectively. In Figure 10 and Figure 11, we also plot the MOS values of fairness at terminals 1 and 2, respectively. In the figures, we show only results of assessment 2 . We do not show results of assessment 1 in this paper since they had similar tendencies to those in assessment 2. The reader is referred to [24] for results of assessment 1 . The $95 \%$ confidence intervals are also plotted in the figures.

In Figure 8, we see that the MOS values of operability for all the values of delay 2 at terminal 1 decrease as local lag 1 increases as in the same delay case. This is because the local information at terminal 1 is buffered for a time of local lag 1; thus, since the interactivity is degraded, each subject feels that the balloon becomes harder and more slippery, and it is difficult to burst the balloon. From Figure 9, we find that the MOS values at terminal 2 hardly depend on local lag 1, and they depend on mainly delay 2 or local lag 2 . This is because the local information at terminal 2 is buffered for a time of delay 2.

In Figure 10 and Figure 11, we notice that the MOS values of fairness for all the values of delay 2 increase as local lag 1 increases. This is because each subject feels fairness strongly when the absolute difference of local lags 1 and 2 becomes smaller.

By comparing Figure 8 and Figure 10, we confirm that there is a trade-off relationship between the MOS value of operability and that of fairness at terminal 1 ; that is, as local lag 1 increases, the MOS value of operability becomes smaller, but that of fairness becomes larger. Also, from Figure 9 and Figure 11, we note that the MOS value of operability at terminal 2 hardly change, but the MOS value of fairness becomes larger.

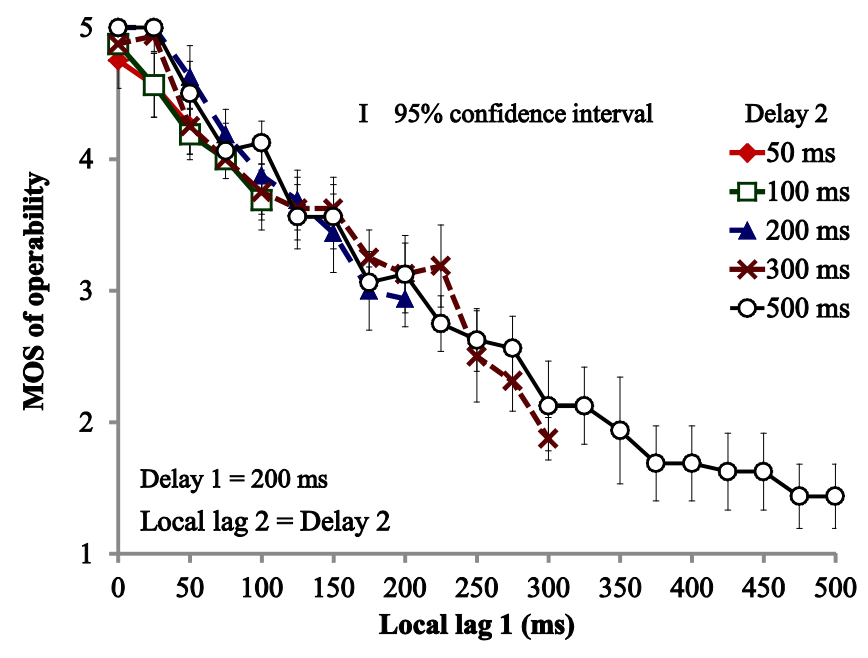

Figure 8. MOS of operability at terminal 1 in different delay case. 


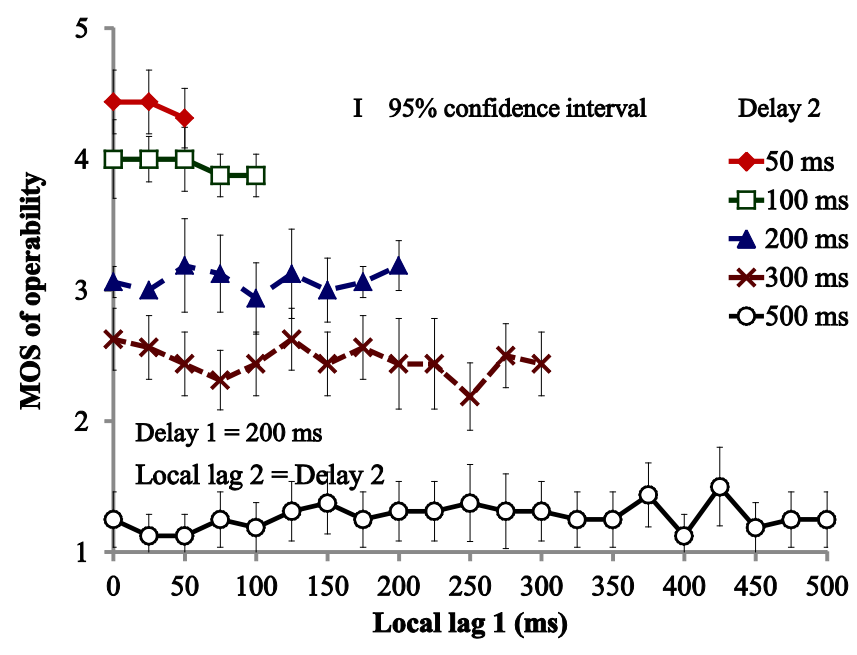

Figure 9. MOS of operability at terminal 2 in different delay case.

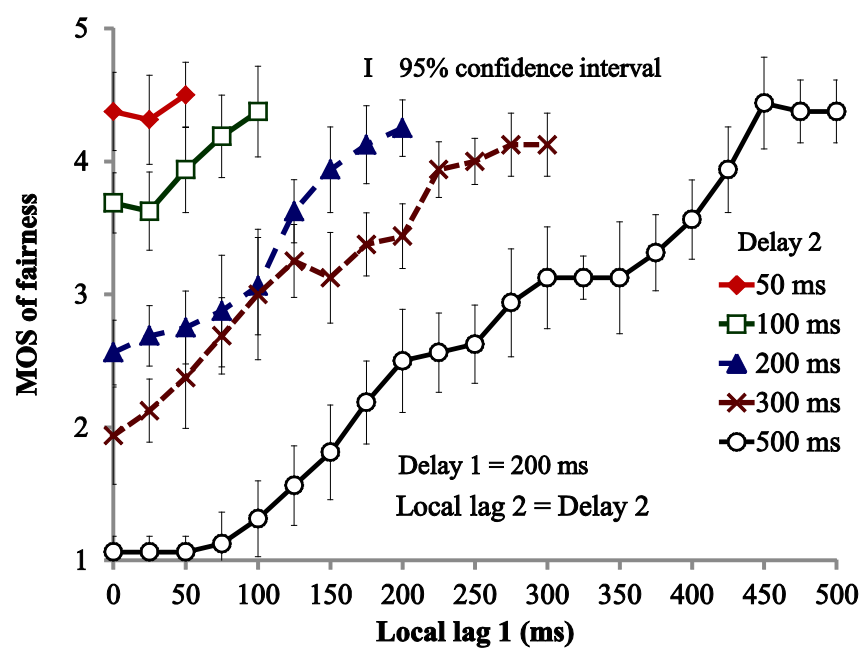

Figure 10. MOS of fairness at terminal 1 in different delay case.

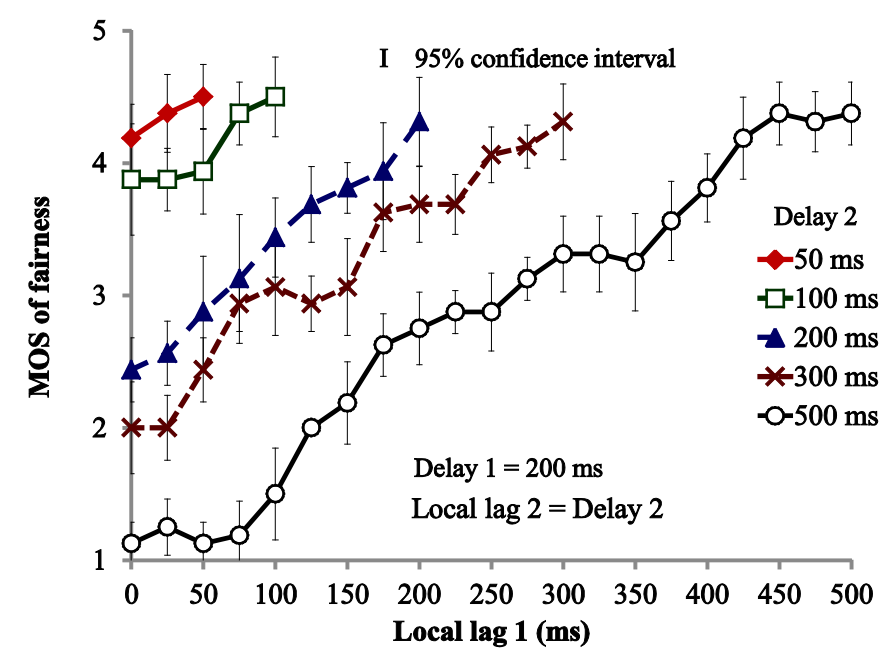

Figure 11. MOS of fairness at terminal 2 in different delay case. 
To clarify the optimum values of local lag at both terminals, we have calculated the average MOS values of two terminals, which are shown in Figure 12 and Figure 13. In Figure 12, we see that the average MOS value of operability for two terminals decreases as local lag 1 becomes larger for each value of delay 2 . In the figure, we also find that delay 2 of $50 \mathrm{~ms}$ has the highest MOS value, and delay 2 of $500 \mathrm{~ms}$ has the lowest for each value of local lag 1. In Figure 13, we notice that the average MOS values increase as local lag 1 becomes larger for all the values of delay 2. From the average MOS values of the two terminals, we further confirm that the trade-off relationship exists between the MOS value of operability and that of fairness. From Figure 13, we also observe that the MOS value is larger than 3.5 when the absolute value of local lag difference (local lag 1 minus local lag 2) is smaller than or equal to around $75 \mathrm{~ms}$. Thus, the allowable range is around $75 \mathrm{~ms}$ according to [23]. This information of allowable range of difference in local lag difference may be helpful for networked applications in which only fairness is focused to be maintained. In our previous work, where we set local lags 1 and 2 to the same value of delays 1 and 2, respectively, we have clarified that the allowable range of absolute difference in network delay is less than or equal to around $75 \mathrm{~ms}$ [6] [22].

We show the MOS values of comprehensive quality at terminals 1 and 2 in Figure 14 and Figure 15, respectively. The average MOS value of comprehensive quality is also plotted in Figure 16. Figure 14 reveals that the

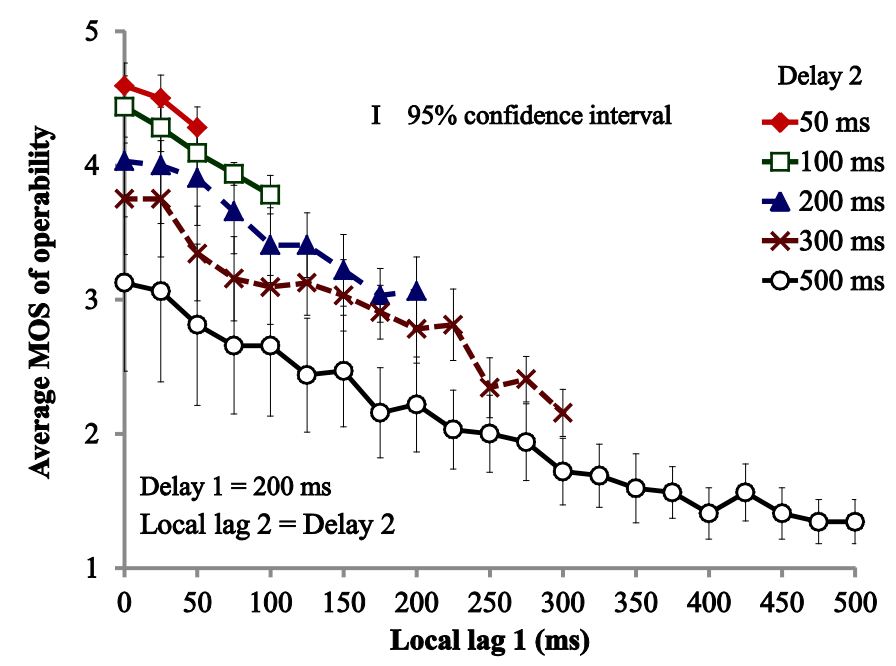

Figure 12. Average MOS of operability for two terminals in different delay case.

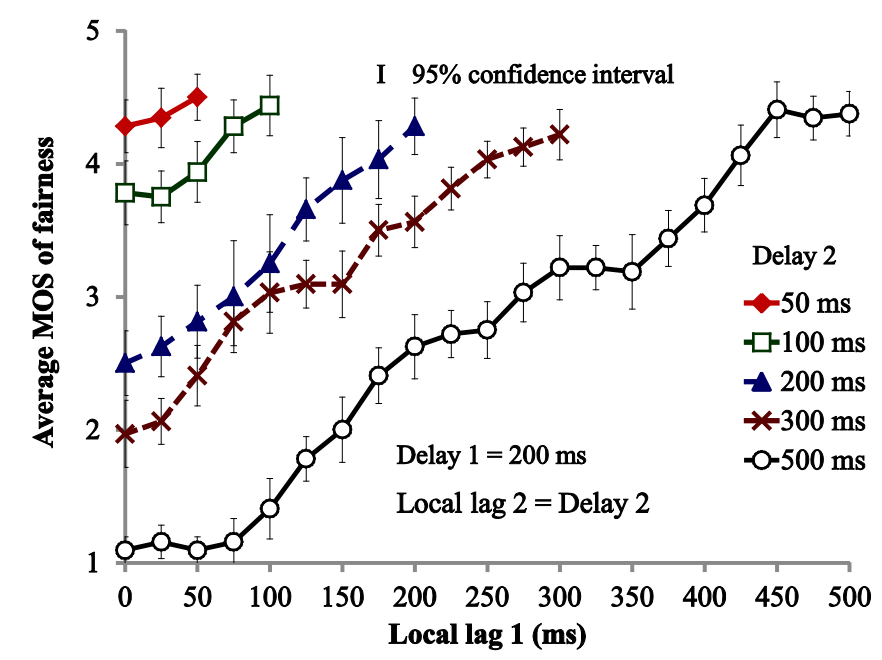

Figure 13. Average MOS of fairness for two terminals in different delay case. 


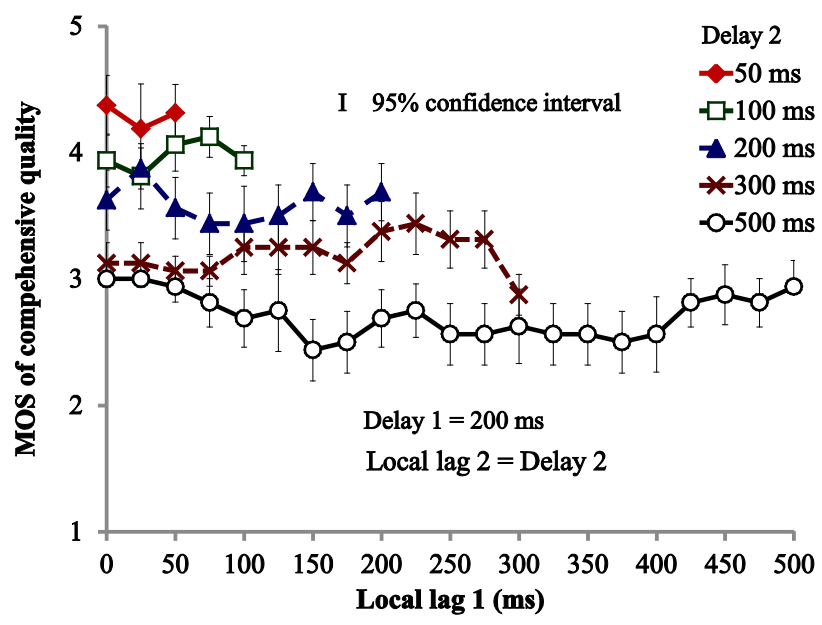

Figure 14. MOS of comprehensive quality at terminal 1 in different delay case.

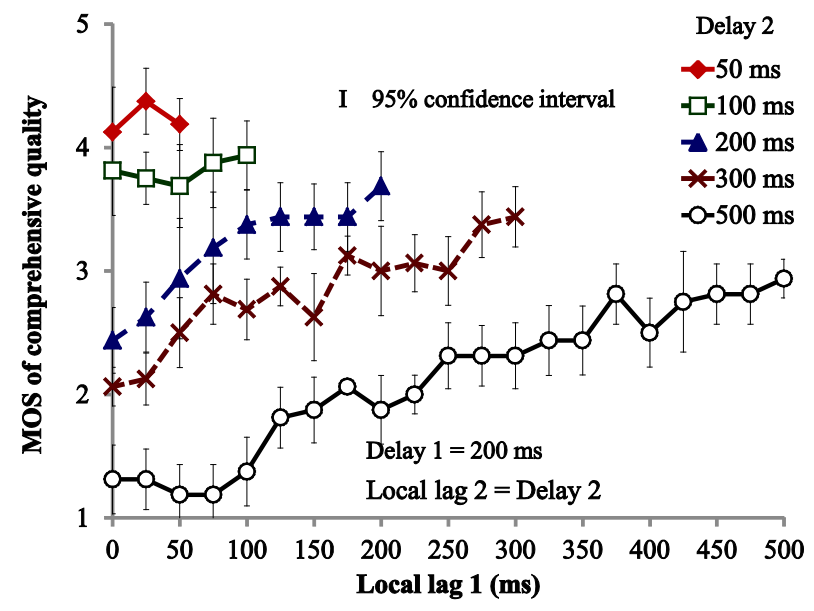

Figure 15. MOS of comprehensive quality at terminal 2 in different delay case.

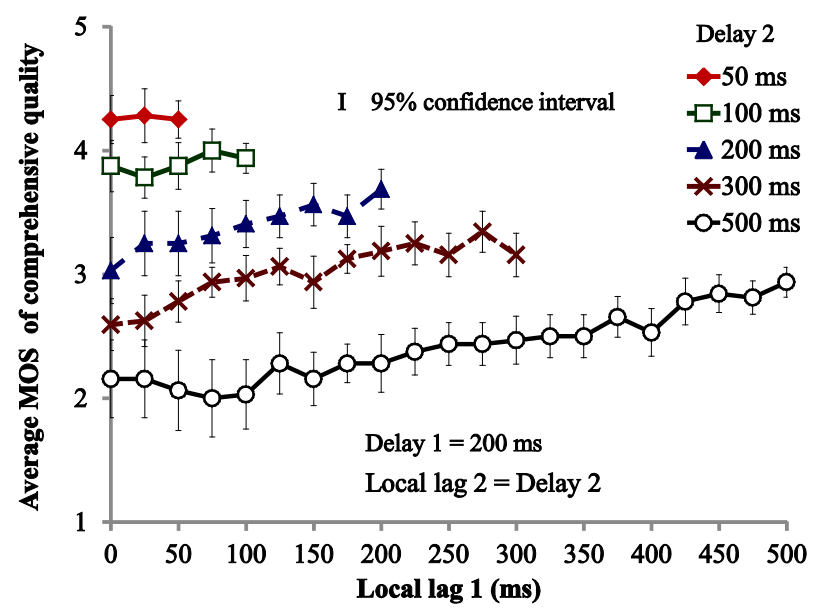

Figure 16. Average MOS of comprehensive quality in different delay case. 
MOS values of comprehensive quality for all the values of delay 2 at terminal 1 hardly depend on local lag 1 . In Figure 15, we find that the MOS value hardly depends on local lag 1 when delay 2 is $50 \mathrm{~ms}$ or $100 \mathrm{~ms}$. For the other values of delay 2, the MOS values increase as local lag 1 becomes larger. From Figure 16, we note that the average MOS value for delay 2 of $50 \mathrm{~ms}$ hardly depends on local lag 1 . For the other values of delay 2, the average MOS values tend to increase slightly as local lag 1 becomes larger. When delay 2 is $100 \mathrm{~ms}$, the optimum value of local lag 1 is from about $75 \mathrm{~ms}$ to around $100 \mathrm{~ms}$. When delay 2 is $200 \mathrm{~ms}, 300 \mathrm{~ms}$ or $500 \mathrm{~ms}$, the optimum value of local lag 1 is within the range from about $125 \mathrm{~ms}$ to around $200 \mathrm{~ms}$, from about $225 \mathrm{~ms}$ to around $300 \mathrm{~ms}$, or from about $425 \mathrm{~ms}$ to around $500 \mathrm{~ms}$, respectively.

From Figure 14 and Figure 15, we notice that the contribution of the fairness is larger than that of the operability to the comprehensive quality. From Figure 16, we can also clarify how the local lags should beset to maintain the comprehensive quality as high as possible at both terminals. Local lags 1 and 2 should be set to the same values of delays 1 and 2, respectively, if the difference in network delays between the terminals is smaller than or equal to about $50 \mathrm{~ms}$. When the difference is larger than about $50 \mathrm{~ms}$, for simplicity, the local lags can be set to the larger value of network delays between the terminals as in the adaptive $\Delta$-causality control.

\subsubsection{Objective Assessment Results}

We show the average numbers of burst balloons at terminals 1 and 2 in Figure 17 and Figure 18, respectively. The average difference in the number of burst balloons is also shown in Figure 19. The 95\% confidence intervals are also plotted in the figures.

In Figure 17, we see that the average number of burst balloons at terminal 1 becomes smaller as local lag 1 increases for each value of delay 2. In Figure 18, we find that the average number of burst balloons at terminal 2 hardly depends on local lag 1. By comparing Figure 17 and Figure 18 with Figure 8 and Figure 9, respectively, we can say that the MOS value of operability decreases as the average number of burst balloons becomes smaller for each value of delay 2. From Figure 19, we notice that the average difference in the number of burst balloons becomes smaller as local lag 1 increases for each value of delay 2 . This is because the local lag difference between the two terminals becomes smaller as local lag 1 increases. By comparing Figure 19 to Figure 10 or Figure 11, we see that the tendencies of the curves are inverses of each other; that is, the highest MOS value can be obtained when the average difference in the number of burst balloons is the smallest for each value of delay 2 .

\subsubsection{Relations between Objective and Subjective Results}

As described in Section 4.2.2, by comparing the objective results to subjective results, we found that they are related to each other. We should clarify how they are related to each other in detail, and examine whether the subjective results can be estimated from the objective results or not. To investigate the relationship between the average number of burst balloons (or the local lag) and the MOS value of operability, we carried out the regression analysis [25]. As a result, we obtained estimated equations shown in Table 1 for both terminals of the two assessments. In Table 1, $O_{\text {MOS }}$ denotes the estimated MOS value of operability, $N_{\text {burst }}$ is the average number of

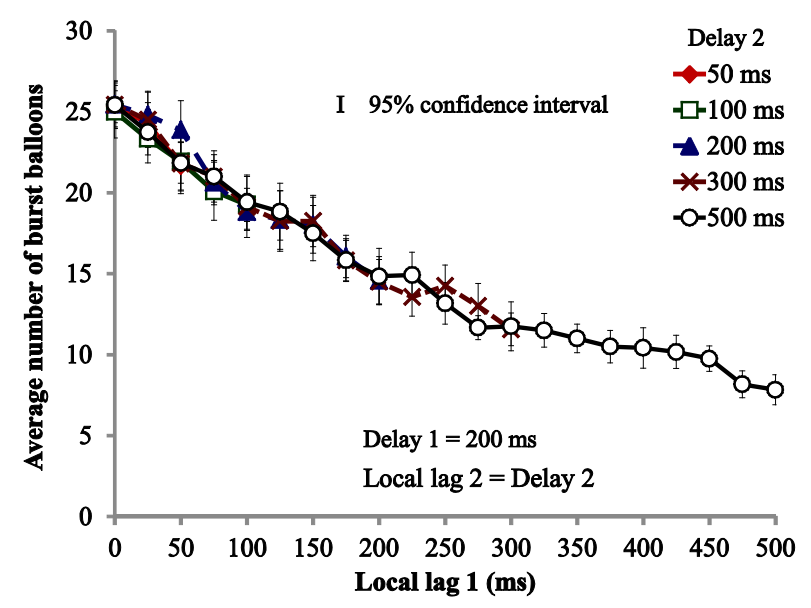

Figure 17. Average number of burst balloons at terminal 1 . 


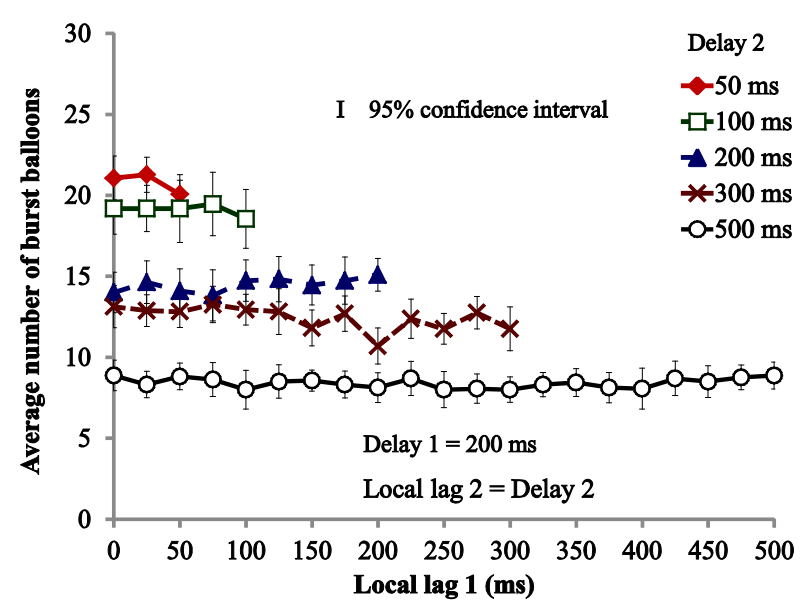

Figure 18. Average number of burst balloons at terminal 2.

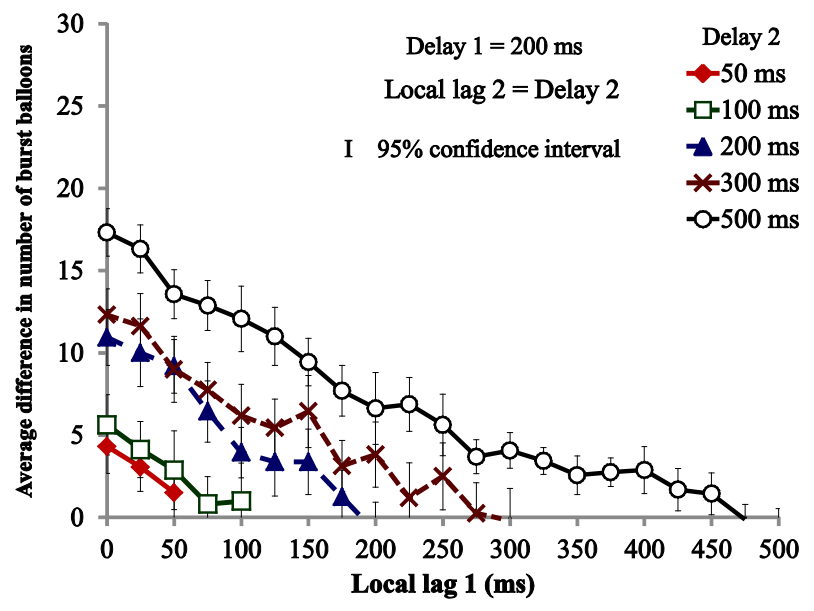

Figure 19. Average difference in number of burst balloons between terminals.

Table 1. Estimated equations for MOS of operability.

\begin{tabular}{cc}
\hline Equation & $\mathbf{R}^{2}$ \\
\hline$O_{\mathrm{MOS}}=0.228 N_{\text {burst }}-0.448$ & 0.968 \\
$O_{\mathrm{MOS}}=-0.007 \Delta+4.634$ & 0.968 \\
\hline
\end{tabular}

burst balloons, $\Delta$ is the local lag, and $R^{2}$ is the contribution rate adjusted for degrees of freedom [25], which shows goodness of fit with the estimated equation. Since the contribution rates are very high in Table 1 , we can say that the MOS value of operability can be estimated with a high degree of accuracy from the average number of burst balloons or the local lag.

We also carried out regression analysis to investigate the relationship between the MOS value of fairness and the absolute value of average difference in the number of burst balloons, and that between the MOS value of fairness and the absolute value of local lag difference. As a result, we obtained equations shown in Table 2 for both terminals in the two cases. In Table $2, F_{\text {Mos }}$ is the estimated MOS value of fairness, $D_{N_{b u r s}}$ is the average difference in the number of burst balloons, and $D_{\Delta}$ is the local lag difference. From Table 2, we find that the contribution rates are high. Thus, we can say that the MOS value of fairness can be estimated with a high degree of accuracy from the absolute value of average difference in the number of burst balloons or the absolute value of the local lag difference. Furthermore, according to high contribution rates shown in Table 3 , where $C_{\text {Mos }}$ is the estimated MOS value of comprehensive quality, we can say that we can estimate the MOS value of compre- 
hensive quality with a high degree of accuracy from the average number of burst balloons and the absolute value of average difference in the number of burst balloons, or from the lcoal lag and the absolute value of local lag difference.

In addition, we examined the relationship between the average MOS values of two terminals and the objective results. The estimated equations for average MOS value of operability are shown in Table 4, where $A_{\text {OMOS }}$ is the estimated average MOS value of operability, $N_{\text {burst } 1}$ is the average number of burst balloons at terminal 1 , and $N_{\text {burst } 2}$ is the average number of burst balloons at terminal 2. From Table 4, we notice that the averge MOS value of operability can be estimated from the average number of burst balloons at two terminals to a large extentsince the contribution rates in the table are high. We also found that the estimated equations for average MOS value of fairness are almost the same as those in Table 2. This means that the average MOS value of fairness can be estimated with a high degree of accuracy from the absolute value of average difference in number of burst balloons or the absolute value of the local lag difference. The estimated equations for average MOS value of comprehensive quality are shown in Table 5 , where $A_{\mathrm{CMOS}}$ is the average MOS value of comprehensive quality, $\Delta_{1}$ is thelocal lag at terminal 1 , and $\Delta_{2}$ is the local lag at terminal 2. According to the high contribution rates shown in Table 5, we can say that the average MOS value of comprehensive quality can be estimated from the average number of burst balloons at two terminals and the absolute value of average difference in the number of burst balloons, or from the lcoal lags at two terminals.

\section{Conclusions}

In this paper, we investigated the influences of network delay on QoE such as the operability and fairness for a balloon bursting game in a networked virtual environment with haptic sense by carrying out subjective and objective QoE assessments. As a result, we found that the operability of haptic interface device strongly depended on the local lag. On the other hand, we can say that the operability depends on the network delay from the local terminal to the other terminal. We also observed that the soft objects became harder and more slippery as the

Table 2. Estimated equations for MOS of fairness.

\begin{tabular}{cc}
\hline Equation & $\mathbf{R}^{2}$ \\
\hline$F_{\text {MOS }}=-0.211\left|D_{N_{\text {bux }}}\right|+4.356$ & 0.891 \\
$F_{\text {MOS }}=-0.007\left|D_{\Delta}\right|+4.315$ & 0.936 \\
\hline
\end{tabular}

Table 3. Estimated equations for MOS of comprehensive quality.

\begin{tabular}{cc} 
Equation & $\mathbf{R}^{2}$ \\
\hline$C_{\text {MOS }}=0.112 N_{\text {burst }}-0.107\left|D_{N_{\text {bus }}}\right|+1.891$ & 0.931 \\
$C_{\text {MOS }}=-0.003 \Delta-0.003\left|D_{\Delta}\right|+4.196$ & 0.928 \\
\hline
\end{tabular}

Table 4. Estimated equations for average MOS of operability.

\begin{tabular}{cc} 
Equation & $\mathbf{R}^{2}$ \\
\hline$A_{\mathrm{O}_{\mathrm{MOS}}}=0.106 N_{\text {bust1 }}+0.125 N_{\text {burst } 2}-0.515$ & 0.977 \\
$A_{\mathrm{O}_{\mathrm{MOS}}}=-0.004 \Delta_{1}-0.003 \Delta_{2}+4.590$ & 0.978 \\
\hline
\end{tabular}

Table 5. Estimated equations for average MOS of comprehensive quality.

\begin{tabular}{cc} 
Equation & $\mathbf{R}^{2}$ \\
\hline$A_{C_{\mathrm{MOS}}}=0.022 N_{\text {burst } 1}+0.106 N_{\text {burst } 2}-0.071\left|D_{N_{\text {bust }}}\right|+1.679$ & 0.966 \\
$A_{C_{\text {MOS }}}=0.001 \Delta_{1}-0.004 \Delta_{2}+4.180$ & 0.956 \\
\hline
\end{tabular}


network delay increased. Also, we examined that the fairness between players depended on the difference in network delay between the terminals or the local lag difference between the terminals. We further confirmed that there existed a trade-off relationship between the operability and fairness. Moreover, we clarified how the local lags should be set at each terminal to be able to keep both operability and fairness as follows. The local lag at each terminal can be set to the same value as the network delay from the local terminal to the other terminal when the absolute difference in network delay between the terminals is smaller than or equal to about $50 \mathrm{~ms}$. When the difference is larger than about $50 \mathrm{~ms}$, we can set the local lags to the larger value of network delay between the terminals.

Additionally, we examined the relationships between subjective and objective assessment results. We found that the MOS value of operability could be estimated from the average number of burst balloons or local lag with a high degree of accuracy. We also illustrated that the MOS value of fairness could roughly be estimated from the average difference in number of burst balloons or the absolute value of local lag difference. Furthermore, we noted that the MOS value of comprehensive quality could be estimated from the average number of burst balloons and the absolute value of average difference in number of burst balloons, or from the local lag and the absolute value of local lag difference to a large extent.

As our future work, we will carry out QoE assessments with other judgments of bursting balloons in the balloon bursting game; for example, a balloon can be burst when the force applied to the balloon reaches a threshold. We will also confirm the trade-off relationship of the operability and fairness in other networked real-time games. We need to confirm whether the MOS value of comprehensive quality can be kept high or not at both terminals by setting the local lags according to the difference in network delay between the terminals as described in Section 4.2.1. Furthermore, it is important to carry out the confirmation in networked real-time games over the Internet.

\section{Acknowledgements}

This work was partly supported by JSPS KAKENHI Grant Number 26420354.

\section{References}

[1] Morris, D., Joshi, N. and Salisbury, K. (2004) Haptic Battle Pong: High-Degree-of-Freedom Haptics in a Multiplayer Gaming Environment. Proceedings of Experimental Gameplay Workshop, Game Developers Conference, March 2004.

[2] Andrews, S., Mora, J., Lang, J. and Lee, W.S. (2006) HaptiCast: A Physically-Based 3D Game with Haptic Feedback. Proceedings of Future Play Conference, October 2006.

[3] Ishibashi, Y. and Kaneoka, H. (2005) Fairness among Game Players in Networked Haptic Environments: Influence of Network Latency. Proceedings of IEEE International Conference on Multimedia and Expo (ICME), 6-8 July 2005. http://dx.doi.org/10.1109/icme.2005.1521359

[4] Ishibashi, Y., Hoshino, S., Zeng, Q., Fukushima, N. and Sugawara, S. (2014) QoE Assessment of Fairness in Networked Game with Olfaction: Influence of Time It Takes for Smell to Reach Player. Springer's Multimedia Systems Journal (MMSJ), Special Issue on Network and Systems Support for Games, 20, 621-631.

[5] Mauve, M., Vogel, J. and Effelsberg, W. (2004) Local Lag and Timewrap: Providing Consistency for Replicated Continuous Applications. IEEE Transactions on Multimedia, 6, 47-57. http://dx.doi.org/10.1109/TMM.2003.819751

[6] Sithu, M., Ishibashi, Y., Huang, P. and Fukushima, N. (2015) QoE Assessment of Fairness between Players for Balloon Bursting Game in Networked Virtual Environment with Haptic Sense. IEICE Technical Report, MVE2014-52, 73-78.

[7] ITU-T Rec. E. 800 (2008) Definitions of Terms Related to Quality of Service. International Telecommunication Union.

[8] Brun, J., Safaei, F. and Boustead, P. (2006) Managing Latency and Fairness in Networked Games. Communications of ACM, 49, 46-51. http://dx.doi.org/10.1145/1167838.1167861

[9] Ishibashi, Y., Hashimoto, Y., Ikedo, T. and Sugawara, S. (2007) Adaptive $\Delta$-Causality Control with Adaptive DeadReckoning in Networked Games. Proceedings of the 13th Annual Workshop on Network and Systems Support for Games (NetGames), September 2007, 75-80. http://dx.doi.org/10.1145/1326257.1326271

[10] ITU-T Rec. P. 10/G. 100 Amendment 1 (2007) New Appendix I-Definition of Quality of Experience (QoE). International Telecommunication Union, January 2007.

[11] Fujimoto, M. and Ishibashi, Y. (2004) The Effect of Stereoscopic Viewing of a Virtual Space on a Networked Game Using Haptic Media. Proceedings of ACM SIGCHI International Conference on Advances in Computer Entertainment 
Technology (ACE), June 2004, 317-320. http://dx.doi.org/10.1145/1067343.1067389

[12] http://geomagic.com/en/products/phantom-omni/overview.

[13] Nakano, S., Maeda, Y., Ishibashi, Y., Fukushima, N., Huang, P. and Psannis, K. (2015) Influence of Network Delay on Fairness between Players in Networked Game with Olfactory and Haptic Senses. IEICE Technical Report, CQ2014-94, 47-52.

[14] Kawano, Y., Hanawa, D. and Yonekura, T. (2004) An Approach to the P2P Type Real-Time Network Virtual Ball-Game with the Mutual Prediction Protocol and AtoZ. Transactions of the Virtual Reality Society of Japan, 9, 141150. (In Japanese)

[15] Kawano, Y. and Yonekura, T. (2006) Realization of Avoidance of the Critical Case in P2P Type Virtual Ball Game by Count Down Protocol. IEICE Transactions on Information and Systems (Japanese Edition), J89-D, 2219-2228.

[16] Kusunose, Y., Ishibashi, Y., Fukushima, N. and Sugawara, S. (2011) QoE Comparison of Competition Avoidance Methods for Management of Shared Object in Networked Real-Time Game with Haptic Media. Proceedings of the 21st International Conference on Artificial Reality and Telexistence (ICAT), Osaka, 28-30 November 2011.

[17] SensAble Technologies, Inc. (2009) OpenHaptics Toolkit Programmer’s Guide. Version 3.0.

[18] Sithu, M., Huang, P., Ishibashi, Y. and Fukushima, N. (2014) Influence of Network Delay on QoE for Soft Objects in Networked Haptic Virtual Environment. IEICE Technical Report, CQ2014-82, 65-70.

[19] Carson, M. and Santay, D. (2003) NIST Net-A Linux-Based Network Emulation Tool. ACM SIGCOMM Computer Communication Review, 33, 111-126. http://dx.doi.org/10.1145/956993.957007

[20] Sithu, M., Ishibashi, Y. and Fukushima, N. (2014) Effects of Dynamic Local Lag Control on Sound Synchronization and Interactivity in Joint Musical Performance. ITE Transactions on Media Technology and Applications, Special Section on Multimedia Transmission System and Services, 2, 299-309.

[21] ITU-R BT. 500-12. Methodology for the Subjective Assessment of the Quality of Television Pictures. International Telecommunication Union, September 2009.

[22] Sithu, M., Ishibashi, Y., Huang, P. and Fukushima, N. (2015) QoE Assessment of Operability and Fairness for Soft Objects in Networked Real-Time Game with Haptic Sense. Proceedings of the 21st Asia-Pacific Conference on Communications (APCC), Kyoto, 14-15 October 2015.

[23] ITU-R BT. 1359-1. Relative Timing of Sound and Vision for Broadcasting, 1998.

[24] Sithu, M., Ishibashi, Y., Huang, P. and Fukushima, N. (2015) Trade-Off Relationship between Operability and Fairness in Networked Balloon Bursting Game Using Haptic Interface Devices. Proceedings of the 9th International Conference on Genetic and Evolutionary Computing (ICGEC), Yangon, 26-28 August 2015.

[25] Chatterjee, S., Hadi, A.S. and Price, B. (1999) Regression Analysis by Example. John Wiley \& Sons, Hoboken. 